\title{
IDH1/2 mutation is a prognostic marker for survival and predicts response to chemotherapy for grade II gliomas concomitantly treated with radiation therapy
}

\author{
YOSHIKO OKITA $^{1}$, YOSHITAKA NARITA ${ }^{1}$, YASUJI MIYAKITA ${ }^{1}$, MAKOTO OHNO $^{1}$, \\ YUKO MATSUSHITA ${ }^{1}$, SHINTARO FUKUSHIMA ${ }^{2}$, MINAKO SUMI ${ }^{3}$, \\ KOICHI ICHIMURA ${ }^{4}$, TAKAMASA KAYAMA ${ }^{1}$ and SOICHIRO SHIBUI ${ }^{1}$
}

\begin{abstract}
Departments of ${ }^{1}$ Neurosurgery and Neuro-Oncology, ${ }^{2}$ Pathology and Clinical Laboratories and ${ }^{3}$ Radiation Oncology,
${ }^{4}$ Division of Brain Tumor Translational Research, National Cancer Center, Tokyo 104-0045, Japan
\end{abstract}

Received April 27, 2012; Accepted June 29, 2012

DOI: 10.3892/ijo.2012.1564

\begin{abstract}
Reliable prognostic biomarkers of grade II gliomas remain unclear. This study aimed to examine the role of mutations of isocitrate dehydrogenase (IDH1/2), 1p/19q co-deletion, and clinicopathological factors in patients with grade II glioma who were primarily treated with radiotherapy or chemoradiotherapy after surgery. Seventy-two consecutive patients, including 49 cases of diffuse astrocytomas (DA), 4 oligodendrogliomas (OL) and 19 oligoastrocytomas (OA), who underwent treatment from 1991 to 2010 at a single institution were examined. The overall survival (OS) of the DA patients (8.3 years) was significantly shorter than that of the OL and OA patients (11.7 years). IDH1/2 mutations were found in $46.9 \%$ of the DA patients and $82.6 \%$ of the OL and OA patients. The progression-free survival (PFS) and OS of the patients with IDHI/2 mutations ( 8.4 and 16.3 years) were significantly longer than those of the patients without $I D H 1 / 2$ mutations (3.3 and 4.5 years). Among the patients with $I D H 1 / 2$ mutations, those who were initially treated with chemoradiotherapy including nimustine hydrochloride (ACNU), had significantly longer PFS than those treated with radiotherapy alone, whereas no significant difference in PFS was observed between the chemoradiotherapy and radiotherapy groups in the patients without IDH1/2 mutations. Oligodendroglial tumors, age $<40$ years, initial Karnofsky performance status (KPS) $\geq 80$, and IDHI/2 mutations were favorable prognostic factors regarding PFS and OS. IDH1/2 mutation was a predictive factor of response to chemoradiotherapy in grade II gliomas. Patients with $I D H I / 2$
\end{abstract}

Correspondence to: Dr Yoshitaka Narita, Department of Neurosurgery and Neuro-Oncology, National Cancer Center Hospital, 5-1-1 Tsukiji, Chuo-ku, Tokyo 104-0045, Japan

E-mail: yonarita@ncc.go.jp

Key words: grade II gliomas, isocitrate dehydrogenase 1, isocitrate dehydrogenase 2, 1p/19q codeletion, chemoradiotherapy, diffuse astrocytoma mutations may benefit more from chemoraiotherapy than those without $I D H 1 / 2$ mutations.

\section{Introduction}

World Health Organization (WHO) grade II gliomas (low-grade gliomas) are slow-growing tumors that include several subtypes, such as diffuse astrocytomas, mixed oligoastrocytomas, and oligodendrogliomas (1). The 10- and 20-year survival rates for patients with grade II glioma are reported to be 48 and $22 \%$ (2), reflecting the malignant potential of these tumors in longterm survival. Radiotherapy is often the treatment of choice for patients with incompletely resected grade II gliomas. However, the timing of radiotherapy for patients with these malignancies remains controversial and no difference in overall survival (OS) between groups receiving early and delayed radiation has been reported (3). Moreover, the efficacy of chemoradiotherapy for grade II gliomas is largely unknown. The addition of procarbazine, lomustine, and vincristine (PCV) therapy to radiotherapy for grade II gliomas conferred a significant increase in OS and progression-free survival (PFS) of $>2$ years in the Radiation Therapy Oncology Group (RTOG) 9802 study (4), suggesting that chemoradiotherapy might be effective for a subset of these patients.

Several studies have attempted to identify prognostic factors for grade II gliomas. To date, older age, astrocytic histology, the presence of neurologic deficits before surgery, larger tumor diameters, and tumors crossing the midline have been proposed as unfavorable prognostic factors (5-9). Several genetic markers, such as $1 \mathrm{p} / 19 \mathrm{q}$ codeletion or mutations of the isocitrate dehydrogenase 1 and 2 genes (IDH1/2), have also been associated with patient survival. Oligodendrogliomas typically show $1 \mathrm{p} / 19 \mathrm{q}$ codeletion $(\leq 70 \%)(10,11)$, and its presence is reported to predict longer survival in oligodendroglial tumors (12). The $1 p / 19 q$ codeletion is also a statistically significant predictor of prolonged survival in patients with astrocytomas (13). Furthermore, 1p/19q codeletion was associated with longer survival in all types of adult gliomas, independent of age and diagnosis $(14,15)$. On the other hand, 1p/19q codeletion did not appear to be a sensitive prognostic biomarker in patients with either grade II astrocytic 
or oligodendroglial tumors who did not receive radiotherapy or chemotherapy after surgery (16).

Mutations of the IDHI/2 genes are common events in gliomas (17), especially among grade II gliomas, where IDH1 mutations are observed in $70-80 \%$ of cases $(11,17,18)$. Glioblastomas and anaplastic astrocytomas (WHO grade III) with IDH1/2 mutations have more favorable prognoses than those with a wild-type phenotype (17). Several studies have indicated that $I D H 1 / 2$ mutations are significantly associated with positive prognosis and chemosensitivity in low-grade gliomas $(19,20)$, whereas others have reported that IDHI/2 mutations were not associated with prognosis $(21,22)$. Thus, the prognostic or predictive values of these genetic markers in grade II gliomas remain controversial.

In the present study, the clinicopathological factors, including age, Karnofsky performance status (KPS), histology, extent of resection, radiotherapy, chemoradiotherapy, largest tumor diameter, and MIB-1 staining index, as well as IDHI/2 mutations and $1 \mathrm{p} / 19 \mathrm{q}$ codeletion, were analyzed in grade II gliomas and correlated with the clinical course of the patients. Oligodendroglial tumors, age $<40$ years, initial KPS $\geq 80$, and $I D H 1 / 2$ mutations were favorable prognostic factors for PFS and OS. The IDH1/2 mutation was a predictive factor of response to chemoradiotherapy in grade II gliomas.

\section{Materials and methods}

Patients and tissue collections. The data were collected from 72 patients who were found with WHO grade II gliomas at the first surgery. These included 49 diffuse astrocytomas and 23 oligodendroglial tumors, including 4 oligodendrogliomas and 19 oligoastrocytomas (male-female, 40:32; median age, 39.0 years). These consecutive cases were diagnosed and treated between 1991 and 2010 at the National Cancer Center Hospital in Japan. The clinical records of the patients were reviewed, and the data on the extent of tumor resection were obtained from the surgical report. Total or subtotal resection was defined as the removal of $90 \%$ or more of the tumor based on the surgeon's clinical report. Fifty-eight patients $(80.6 \%)$ underwent initial surgeries followed by radiotherapy (22.2\%) or chemoradiotherapy with ACNU (58.3\%). Three patients with total or subtotal removal and 11 with partial resection or biopsy (19.4\%) were followed-up without radiotherapy until progressive disease. Of the remaining patients, those who underwent initial treatment between 1991 and 2006 were treated with chemoradiotherapy and those treated between 2007 and 2010 underwent radiotherapy alone based on our treatment protocols. The radiation doses were 60 Gy before 2006 and 54 Gy after 2007. The chemotherapy in the diffuse astrocytoma cases consisted of ACNU administered twice during radiotherapy and 3 additional doses every 2 months after radiotherapy. The patients with oligodendroglial tumors received ACNU + VCR (vincristine) twice during radiotherapy, and thereafter, PAV [ACNU + VCR + PCZ (procarbazine)] was administered in 3 cycles every 2 months after radiotherapy. Each patient was worked up by MRI every 3-4 months until 2 years from the initial treatment and then every 6 months after 2 years. Progression was determined when the MRI showed a new enhancing lesion with Gd-DTPA, a new high intensity lesion or an obvious increased lesion (at least 20\% larger than previous
MRI in diameter) on T2/FLAIR images. Clinical deterioration of a patient was also determined as progression.

The formalin-fixed paraffin-embedded tumor samples and frozen specimens, when available, were collected from the primary resection for all the patients who underwent surgery in the National Cancer Center and whenever possible for those operated at other hospitals. The samples were examined for IDH1/2 mutations and $1 \mathrm{p} / 19 \mathrm{q}$ codeletion only when sufficient material for DNA extraction was available at either the primary or secondary resection. The study was approved by the internal review board of the National Cancer Center. The detailed information for all the 72 patients is listed in Table I.

Hematoxylin and eosin staining and immunohistochemical staining for MIB-1 and IDHI. The surgical specimens were fixed in $10 \%$ formalin and embedded in paraffin. The hematoxylin and eosin-stained specimens were examined to determine the histological tumor type. The multiple serial sections were subjected to immunohistochemical analyses (IHC) to visualize local staining. Antigen retrieval was carried out by exposing the tissue sections to $15 \mathrm{~min}$ of microwave heating in $0.1-\mathrm{mol} / 1$ sodium citrate (pH 6.0). This was followed with immunostaining of the specimens with the streptavidin-biotin-peroxidase complex method (Vectastain, Vector Laboratories, Inc., Burlingame, CA, USA). The samples were incubated in human monoclonal antibodies against MIB-1 (Dako, Tokyo, Japan). Positive immunostaining results were detected with the diaminobenzidine reaction, and the slides were subsequently counterstained with hematoxylin, dehydrated, cleared, and mounted.

Cell counting was performed with the aid of a light microscope (Olympus Corp., Tokyo, Japan). Cell counting was done at a magnification of $x 400$. At least 200 tumor cells were counted, and the results were expressed as the mean of the counts obtained from 3 different locations within each specimen. The MIB-1-stained cells were also counted, and the percentage of the MIB-1-stained cells was calculated within the observed field and expressed as the MIB-1 index.

Human monoclonal antibodies specific against IDH1R132H and IDH1-R132S were used to identify these 2 types of IDH1 mutations (Medical \& Biological Laboratories, Tokyo, Japan). Positive immunostaining results were detected with the diaminobenzidine reaction, and the slides were subsequently counterstained with hematoxylin, dehydrated, cleared, and mounted. The positive granular cytoplasmic staining of the tumor cells was evaluated for mutant IDHI (23).

Extraction of nucleic acids. The tumor samples were immediately frozen in liquid nitrogen and stored at $-80^{\circ} \mathrm{C}$. A peripheral blood sample was drawn from each patient and stored at $-80^{\circ} \mathrm{C}$. Total DNA was extracted from either frozen tissue samples or paraffin-embedded specimens and from the patients' blood with a DNeasy Blood \& Tissue kit (Qiagen Sciences, Germantown, MD, USA) according to the manufacturer's instructions.

Sequencing of IDHI/2. A 129-base pair (bp) fragment of IDHI containing codon 132 or a 150-bp fragment of $I D H 2$ containing codon 172 was PCR amplified using the forward primer IDH1f (CGGTCTTCAGAGAAGCCATT) and reverse primer IDH1r (GCAAAATCACATTATTGCCAAC) for $I D H I$ and the forward primer IDH2f (AGCCCATCATCTGCAAAAAC) and 
reverse primer IDH2r (CTAGGCGAGGAGCTCCAGT) for IDH2 (18). The thermocycling conditions consisted of $5 \mathrm{~min}$ at $95^{\circ} \mathrm{C}, 35$ cycles for $30 \mathrm{sec}$ at $95^{\circ} \mathrm{C}, 40 \mathrm{sec}$ at $56^{\circ} \mathrm{C}$, and $50 \mathrm{sec}$ at $72^{\circ} \mathrm{C}$, followed by $10 \mathrm{~min}$ at $72^{\circ} \mathrm{C}$. For confirmation, the forward primer IDH1fc (ACCAAATGGCACCATACGA) and reverse primer IDH1rc (TTCATACCTTGCTTAATGGGTGT) generating a 254-bp fragment and the forward primer IDH2fc (GCTGCAGTGGGACCACTATT) and reverse primer IDH2rc (TGTGGCCTTGTACTGCAGAG) generating a 293-bp fragment were used for amplification with the same thermocycling conditions (24). After the purification of the PCR products using the QIAquick PCR Purification kit (Qiagen), DNA sequencing for the IDH1/2 gene was performed with an ABI PRISM 310 Genetic Analyzer (Applied Biosystems), using the same primers as for PCR.

Ip and $19 q$ status by fluorescence in situ hybridization. For fluorescence in situ hybridization (FISH), the tumor sections were deparaffinized in Hemo-De (Falma, Tokyo, Japan), dehydrated with $100 \%$ ethanol, and digested using a Paraffin Pretreatment kit (Vysis-Abbott, Tokyo, Japan) according to the manufacturer's protocol. Each section was hybridized with LSI 1p36/1q25 and LSI 19q13/19p13 probes (Vysis-Abbott). The probes and target DNA were denatured individually at $72^{\circ} \mathrm{C}$ for $5 \mathrm{~min}$, followed by 2 overnight incubations at $37^{\circ} \mathrm{C}$. Posthybridization washes were carried out in standard saline solution twice, and the sections were air-dried. The nuclei were counterstained with 4,6-diamidino2-phenylindole. The sections were analyzed using a fluorescence microscope (Biorevo BZ-9000, Keyence, Japan).

The $1 \mathrm{p}$ or $19 \mathrm{q}$ deletions were considered present when the population of the cells with single $1 \mathrm{p} 36$ or single $19 \mathrm{q} 13$ was $<50 \%$ of the cells with double $1 \mathrm{p} 36$ or double 19p13, respectively. At least 100 non-overlapping nuclei were counted per hybridization.

Ip and $19 q$ status by multiplex ligation-dependent probe amplification analysis. We used the SALSA P088 kit (MRC Amsterdam, The Netherlands) containing $161 \mathrm{p}$ probes (6 probe at 1p36), $819 \mathrm{q}$ probes, and 21 control probes specific to other chromosomes, including 2 probes for $19 \mathrm{p}$. Information regarding the probe sequences and ligation sites can be found at http://www.mlpa.com. Multiplex ligation-dependent probe amplification (MLPA) analysis was performed as described previously $(25,26)$. The $1 \mathrm{p} 36$ or $19 \mathrm{q}$ deletions were considered present when 5 of 6 markers for $1 p 36$ and 5 of 8 markers for $19 q$ in each chromosome arm had normalized ratios $<0.75$.

Statistical analysis. All the statistical analyses, including the Kaplan-Meier survival analysis, were performed using the JMP ver. 8 software (Tokyo, Japan). The multivariate analysis with Cox regression, which was used to assess the independent prognostic factors for all the 72 cases, was performed only for the variables with $\mathrm{p}<0.1$ and which included the data obtained in the univariate analysis for all the patients. A similar analysis was performed for 58 cases with radiotherapy or chemoradiotherapy.

\section{Results}

Progression-free and OS. The PFS and median OS times for all the 72 grade II glioma patients were 5.8 and 10.3 years, respectively (male-female, 40:32; median age, 39.0 years; Table I).
Table I. Characteristics of patients with grade II gliomas.

\begin{tabular}{lccc}
\hline Characteristic & $\begin{array}{c}\text { No. of } \\
\text { patients }\end{array}$ & Years & $\begin{array}{c}\text { Percentage } \\
(\%)\end{array}$ \\
\hline Sex & & \\
Male & 40 & 55.6 \\
Female & 32 & 44.4
\end{tabular}

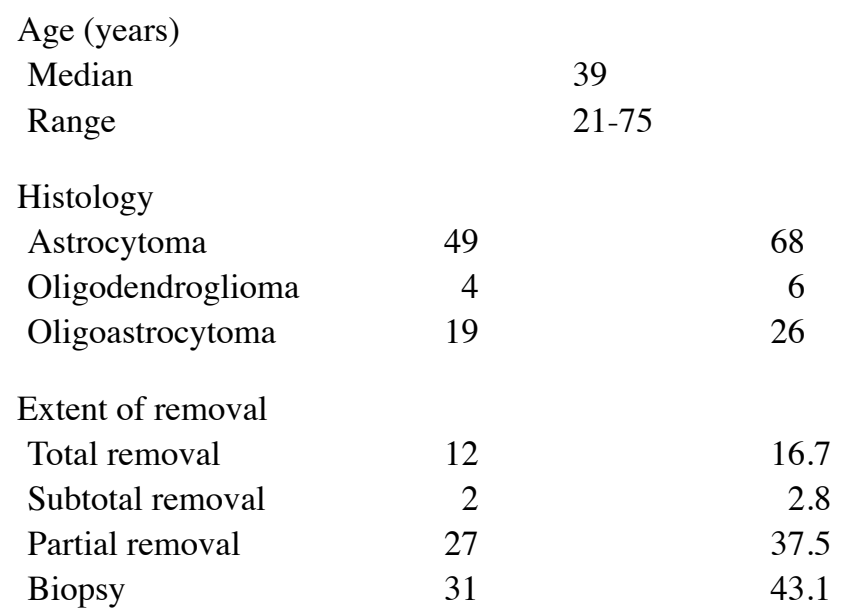

Largest diameter of initial tumor $(\mathrm{cm})$

$<6$

55.6

$\geq 6 \quad 32$

44.4

Initial KPS

$<80$

$\geq 80$

94.4

MIB-1 index (\%)

Median

IDH mutation

Mutation

Wild-type

Loss of $1 \mathrm{p} / 19 \mathrm{q}$

$1 \mathrm{p} / 19 \mathrm{q}$ codeletion $\quad 15$

25.0

$1 \mathrm{p}$ deletion

19q deletion

40.0

38.3

Initial radiotherapy

$+$

80.6

-

Initial chemotherapy

ACNU

44

61

TMZ

3

None

26

36

PFS (years)

Median

Overall survival (years)

Median

10.3 
Table II. Univariate analyses of progression-free survival time and overall survival time of patients with grade II gliomas.

\begin{tabular}{|c|c|c|c|c|}
\hline Variable & $\begin{array}{l}\text { No.of } \\
\text { cases }\end{array}$ & PFS (95\% CI) & $\begin{array}{l}\text { p-value } \\
\text { (log-rank) }\end{array}$ & OS $(95 \% \mathrm{CI})$ \\
\hline
\end{tabular}

\section{Histology}

Astrocytoma

Oligodendroglioma/

49

23

$3.6(2.1-7.7)$

oligoastrocytoma

Age

$<40$ years

38

34

$7.0(3.6-9.3)$

$3.1(1.8-8.9)$

0.2

NR (8.0-NR)

$4.3(3.9-16.3)$

IDH mutation

Mutation

42

30

8.4 (3.2-10.2)

Wild-type

$3.3(1.7-7.0)$

0.04

16.3 (9.6-18.2)

$4.5(3.9-10.0)$

Extent of removal

Total and subtotal removal

14

10.4 (2.5-14.4)

58

$4.3(2.3-8.3)$

Largest diameter of initial

tumor $(\mathrm{cm})$

$$
<6
$$

$\geq 6$

40

32

$7.7(2.3-10.4)$

$4.3(2.1-8.3)$

Initial KPS

$$
<80
$$

$\geq 80$$$
4
$$

$0.6(0.4-8.4)$

$6.8(3.1-8.9)$

0.01

$1.7(0.5-10.3)$

$11.7(8.0-18.2)$

MIB-1 index

$<4 \%$

33

21

$8.1(2.3-8.9)$

4.3 (1.8-NR)

$1 \mathrm{p} / 19 \mathrm{q}$

$1 \mathrm{p} / 19 \mathrm{q}$ codeletion $(+)$

1p/19q codeletion (-)

15

45

6.8 (2.2-NR)

$3.6(2.3-8.4)$

$1 \mathrm{p}$

$1 \mathrm{p}$ deletion

24

36

$5.8(2.5-9.3)$

Intact

$19 \mathrm{q}$

$19 \mathrm{q}$ deletion

23

37

$7.0(4.2-9.3)$

$3.1(1.9-10.2)$

Initial radiotherapy

$+$

58

$4.3(2.9-8.9)$

-

14

$7.7(2.5-9.1)$

Initial treatment

Radiotherapy alone

Chemoradiotherapy

$16 \quad 2.9(0.7-4.3)$

42

$8.1(3.2-10.2)$
0.6

9.6 (5.1-NR)

NR (3.9-NR)

0.4

11.7 (4.3-11.7)

0.2

8.3 (4.4-NR)

0.96

$11.7(4.2-11.7)$

0.9

9.6 (4.4-NR)

0.5

11.7 (4.5-11.7)

0.5

8.3 (3.9-NR)

0.98

8.3 (5.1-18.2)

0.2

$11.7(4.2-16.3)$

0.01

$4.2(2.7-5.1)$

0.0002

NR, PFS or median survival time is not reached; CI, confident interval. 
These patients were initially treated with surgery followed by radiotherapy $(22.2 \%)$ or chemoradiotherapy $(58.3 \%)$. The median follow-up time for all the 72 patients was 6.4 years, and it was 7.6 years for the patients treated with chemoradiotherapy $(n=42)$ and 4.0 years for those who underwent radiotherapy alone $(n=16)$.

Progression-free and OS times according to clinical factors. The univariate analysis (Table II) showed that the patients with oligodendroglial tumors $(n=23)$ had longer OS than those with diffuse astrocytoma $(n=49 ; \mathrm{p}=0.04)$. The PFS and OS were 3.6 and 8.3 years, respectively, in the patients with diffuse astrocytoma, and 8.3 and 11.7 years, respectively, in the patients with oligodendroglioma or oligoastrocytoma (Fig. 1A and B). The patients younger than 40 years $(n=38)$ had longer OS than those who were 40 years or older $(n=34 ; p=0.02)$. The PFS and median survival time of the patients in the younger age groups were 7.0 years and still not reached, respectively, whereas the PFS and OS of the patients in the older age groups were 3.1 and 4.3 years, respectively. The patients with an initial KPS score $\geq 80(n=68)$ had significantly longer OS $(p=0.0006)$ and PFS ( $p=0.01)$ than those with a KPS score $<80(n=4)$. The PFS and OS of the patients with a KPS score $\geq 80$ were 6.8 and 11.7 years, respectively, and those of the patients with a KPS score $<80$ were 0.6 and 1.7 years, respectively. The patients in the total or subtotal resection ( $\geq 90 \%$ removal) groups $(n=14$; median age, 34.0 years) tended to have longer OS than those in the partial $(<90 \%)$ removal or biopsy groups $(n=58$; median age, 41.0; $\mathrm{p}=0.08$ ). The PFS and OS were 10.4 and 18.3 years, respectively, in the patients in the total or subtotal resection groups and 4.3 and 10.0 years, respectively, in the patients in the partial resection or biopsy groups. The patients who were initially treated with chemoradiotherapy after surgery showed significantly longer PFS $(p=0.01)$ and OS $(p=0.0002)$ than those treated with radiotherapy alone (Fig. 1C and D). The PFS and OS of the patients who were initially treated with radiotherapy after surgery $(n=16)$ were 2.9 and 4.2 years, respectively, and the PFS and OS of the patients who were initially treated with chemoradiotherapy after surgery $(n=42)$ were 8.1 and 18.2 years, respectively. According to MIB-1 staining index, there was no significant difference of survival between groups with cut-off point at 4,8 and $15 \%$ in our study.

Presence of $1 p / 19 q$ codeletion, $1 p$ deletion, and $19 q$ deletion and survival. The presence of $1 \mathrm{p} / 19 \mathrm{q}$ deletions was determined for 25 or 26 primary resections and for 7 or 2 secondary resection samples by MLPA or FISH, respectively. The 1p/19q codeletion was observed in $15.9 \%$ (7/44) of the astrocytomas and $50 \%$ $(8 / 16)$ of the oligodendroglial tumors. The OS of the patients with $1 \mathrm{p} / 19 \mathrm{q}$ codeletion was 11.7 years, and the OS of those without $1 \mathrm{p} / 19 \mathrm{q}$ codeletion was 8.3 years $(\mathrm{p}=0.2$; Fig. $1 \mathrm{E}$ and $\mathrm{F}$ ). In the patients with astrocytic tumors, the median survival time of those with $1 \mathrm{p} / 19 \mathrm{q}$ codeletion was not reached and the OS of those without $1 \mathrm{p} / 19 \mathrm{q}$ codeletion was 6.3 years $(\mathrm{p}=0.5)$. The OS of the patients with $1 \mathrm{p} / 19 \mathrm{q}$ codeletion was 11.7 years, and the OS of those without $1 \mathrm{p} / 19 \mathrm{q}$ codeletion was 10.3 years in the oligodendroglial tumors $(p=0.5)$. The presence of $1 p / 19 q$ codeletion, $1 p$ deletion, or $19 q$ deletion was not correlated with the PFS or OS time (Table II).
Table III. Mutation of $I D H 1 / 2$.

Diffuse astro- Oligodendro- Oligoastro-

cytoma $(\%)$ glioma $(\%)$ cytoma $(\%)$

IDH1/2 mutation

by sequence

\begin{tabular}{|c|c|c|c|}
\hline IDH1 R132H & $13(26.5)$ & $2(50.0)$ & $5(26.3)$ \\
\hline IDH1 R132S & $1 \quad(2.0)$ & $\begin{array}{ll}0 & (0.0)\end{array}$ & $\begin{array}{ll}0 & (0.0)\end{array}$ \\
\hline IDH2 R172K & $1 \quad(2.0)$ & $\begin{array}{ll}0 & (0.0)\end{array}$ & $\begin{array}{ll}0 & (0.0)\end{array}$ \\
\hline Wild-type & $15(30.6)$ & $1(25.0)$ & $2(10.5)$ \\
\hline
\end{tabular}

IDH mutation

by IHC

\begin{tabular}{|c|c|c|c|}
\hline IDH1 R132H & $8(16.3)$ & $1(25.0)$ & $11(57.9)$ \\
\hline IDH1 R132S & $\begin{array}{ll}0 & (0.0)\end{array}$ & $\begin{array}{ll}0 & (0.0)\end{array}$ & $\begin{array}{ll}0 & (0.0)\end{array}$ \\
\hline Mutation (-) & $11(22.4)$ & $\begin{array}{ll}0 & (0.0)\end{array}$ & $1 \quad(5.3)$ \\
\hline Total & $49(100)$ & $4(100)$ & $19(100)$ \\
\hline Mutation & $23(46.9)$ & $3(75.0)$ & $16(84.2)$ \\
\hline Wild-type & $26(53.1)$ & $1(25.0)$ & $3(15.8)$ \\
\hline
\end{tabular}

IHC, immunohistochemical staining.

IDH1/2 mutations and survival in the whole series. IDH1/2 mutations were determined in 55 samples at the primary resection and 17 at the secondary resection by IHC alone for 32 cases (44.4\%) and by direct sequencing in 40 cases (55.6\%). IDH1/2 mutations were found in $46.9 \%$ (23/49) of the astrocytomas, $84.2 \%(16 / 19)$ of the oligoastrocytomas, and $75.0 \%(3 / 4)$ of the oligodendrogliomas (Table III).

The patients with $I D H 1 / 2$ mutations $(n=42)$ had longer PFS $(\mathrm{p}=0.04)$ and $\mathrm{OS}(\mathrm{p}=0.004)$ than those without IDH1/2 mutations ( $\mathrm{n}=30$; Table II). The PFS and OS of the patients with IDH1/2 mutations were 8.4 and 16.3 years, respectively, and the PFS and OS of the patients without $I D H 1 / 2$ mutations were 3.3 and 4.5 years, respectively (Fig. $1 \mathrm{G}$ and $\mathrm{H}$ ). The diffuse astrocytoma patients with $I D H 1 / 2$ mutations $(n=23)$ tended to have longer survival times than those without $I D H 1 / 2$ mutations $(\mathrm{n}=26)$, although the difference was not significant $(\mathrm{p}=0.08)$. The median survival time of the diffuse astrocytoma patients with $I D H 1 / 2$ mutations was not reached and that of the diffuse astrocytoma patients without $I D H 1 / 2$ mutations was 4.4 years. The oligodendroglial tumor patients with $I D H 1 / 2$ mutations also tended to have longer, though not significant, survival times $(\mathrm{p}=0.1)$.

The survival of the patients with $I D H 1 / 2$ mutations and $1 \mathrm{p} / 19 \mathrm{q}$ codeletion was longer than that of the patients with neither IDHI/2 mutations nor $1 \mathrm{p} / 19 \mathrm{q}$ codeletion (11.7 vs. 4.4 years, respectively), although the difference did not reach statistical significance $(\mathrm{p}=0.1)$. Furthermore, a combined IDHI/2 and $1 \mathrm{p} / 19 \mathrm{q}$ status did not correlate with the PFS and OS of the patients who were initially treated with chemoradiotherapy after surgery regardless of the histological tumor type. 

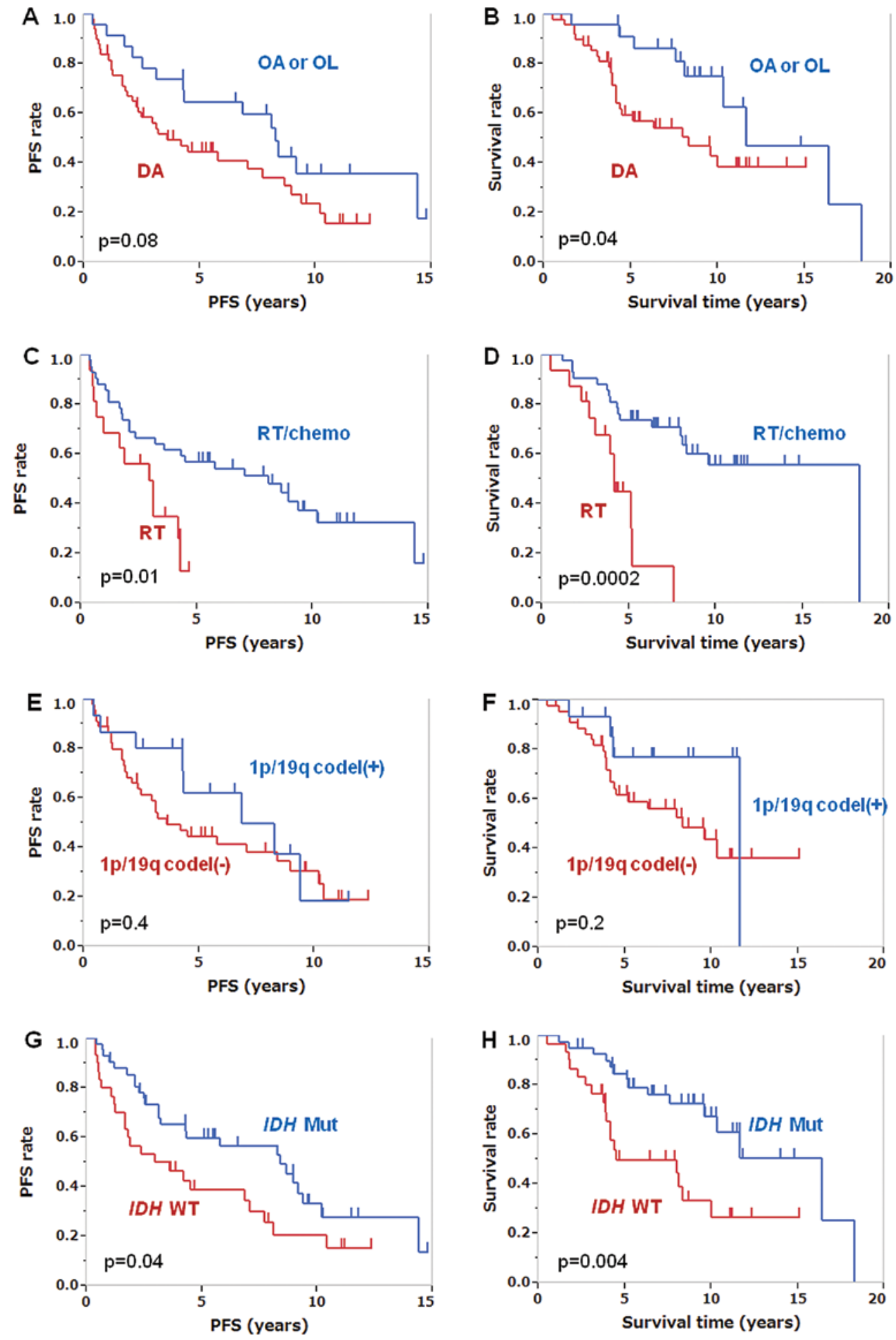

Figure 1. Kaplan-Meier survival curves of the patients with WHO grade II gliomas grouped according to genetic and clinical factors associated with overall survival (OS) and progression-free survival (PFS) by univariate analysis. The survival estimates were calculated according to the following variables: (A) PFS, diffuse astrocytoma (DA) versus oligodendroglial tumors (OA or OL); (B) OS, diffuse astrocytoma (DA) versus oligodendroglial tumors (OA or OL); (C) PFS, radiotherapy (RT) versus chemoradiotherapy (RT/chemo); (D) OS, radiotherapy (RT) versus chemoradiotherapy (RT/chemo); (E) PFS, 1p/19q codeletion (codel) (+) or (-); (F) OS, 1p/19q codeletion (codel) (+) or (-); (G) PFS, IDH1/2 mutation (mut) or wild-type (WT); and (H) OS, IDH1/2 mutation (mut) or wild-type (WT).

In the total or subtotal resection group, the patients with $I D H 1 / 2$ mutations had longer OS than those without $I D H 1 / 2$ mutations ( $\mathrm{p}=0.04$; Fig. $2 \mathrm{~A}$ ). The OS of the patients with $I D H 1 / 2$ mutations ( $\mathrm{n}=6,2$ diffuse astrocytomas, 3 oligoastrocytomas, and 1 oligodendrogliomas) was 18.2 years; to date, 5 are still alive and 1 is dead. The OS of the patients without $I D H 1 / 2$ mutations $(\mathrm{n}=8,7$ astrocytomas and 1 oligoastrocytoma) was 8.0 years. In the partial resection or biopsy group, the patients with $I D H 1 / 2$ mutations had longer OS than those without $I D H 1 / 2$ mutations in the partial resection or biopsy group ( $\mathrm{p}=0.01$; Fig. $2 \mathrm{~B})$. The OS of the patients with $I D H 1 / 2$ mutations ( $\mathrm{n}=36,21$ diffuse astrocytomas, 13 oligoastrocytomas, and 2 oligodendrogliomas) 
A Total or subtotal resection $\pm I D H$ mutation OS

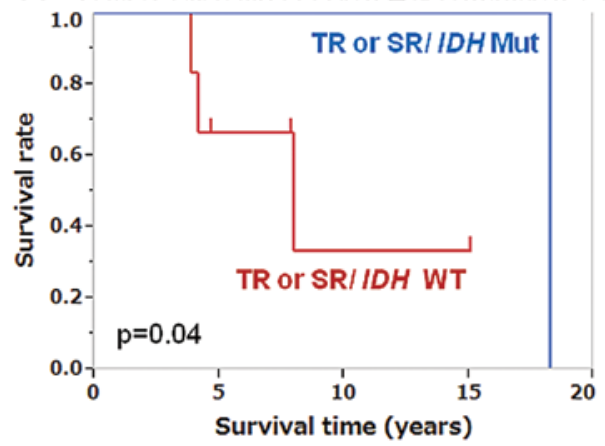

C All histology PFS

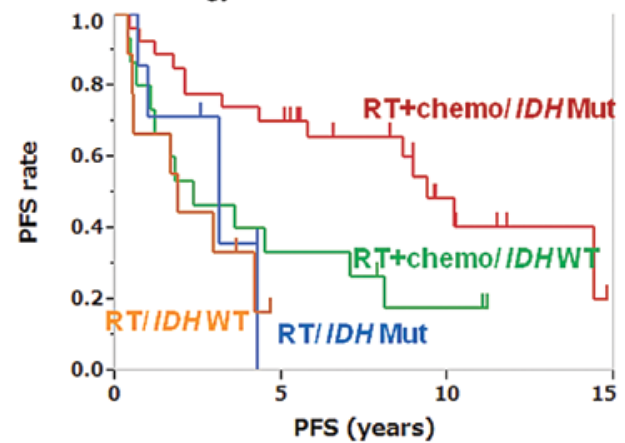

E Astrocytic tumors PFS

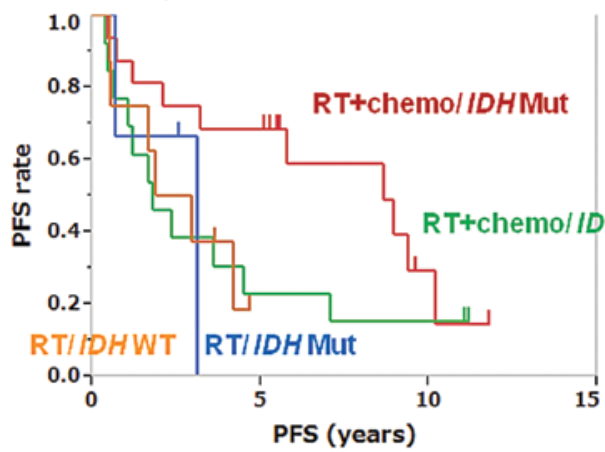

G Oligodendroglial tumors PFS

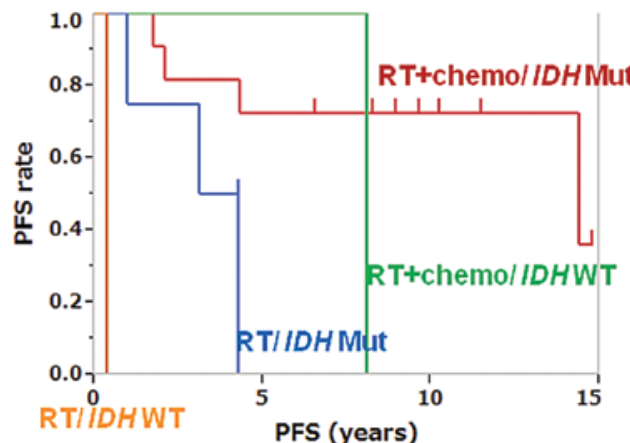

B Partial resection or biopsy $\pm I D H$ mutation OS

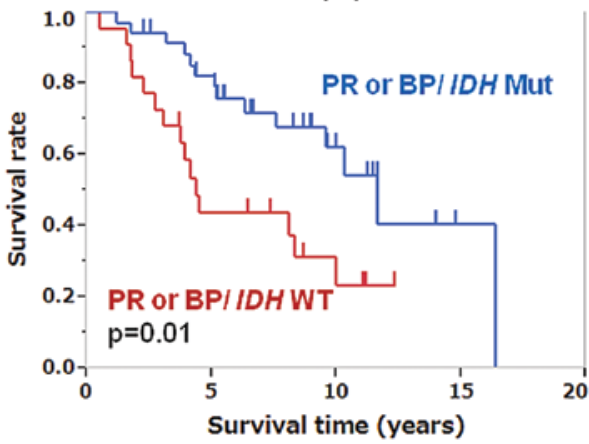

D All histology OS

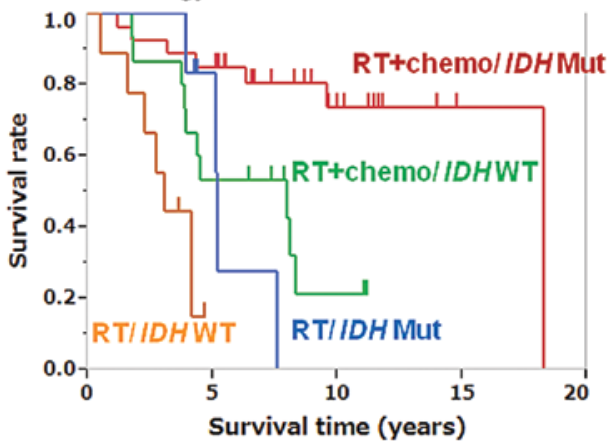

F Astrocytic tumors OS

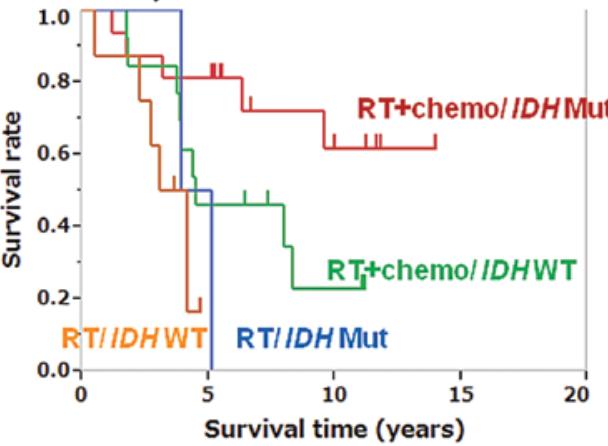

H Oligodendroglial tumors OS

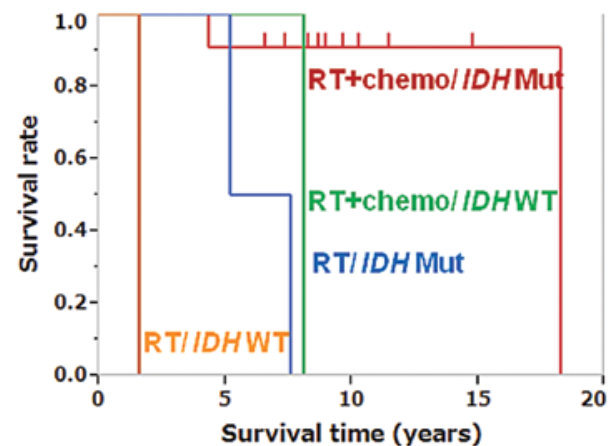

Figure 2. (A and B) Kaplan-Meier survival curves of the patients in the total or subtotal tumor resection (TR or SR) (A) and partial resection or biopsy (PR or BP) (B) groups according to the IDH1/2 status. (C-H) Kaplan-Meier survival curves of the patients who were initially treated with radiotherapy (RT) and chemotherapy (chemo) or radiotherapy alone according to IDH1/2 status associated with the overall survival (OS) and progression-free survival (PFS) by univariate analysis: (C) PFS of all the WHO grade II gliomas, (D) OS of all the WHO grade II gliomas, (E) PFS of the diffuse astrocytomas, (F) OS of the diffuse astrocytomas, (G) PFS of the oligodendroglial tumors, $(\mathrm{H})$ OS of the oligodendroglial tumors.

was 11.7 years, and that of the patients without $I D H 1 / 2$ mutations in these groups $(n=22,19$ diffuse astrocytomas, 2 oligoastrocytomas, and 1 oligodendroglioma) was 4.4 years.
IDH1/2 mutations and survival in the patients who underwent chemoradiotherapy after surgery. Among the grade II glioma patients who were initially treated with chemoradiotherapy 
Table IV. PFS and OS in patients with radiotherapy or chemoradiotherapy according to IDH1/2 status.

\begin{tabular}{|c|c|c|c|c|}
\hline Variable & & No. of cases & PFS (95\% CI) & OS $(95 \% \mathrm{CI})$ \\
\hline \multicolumn{5}{|c|}{ All grade II gliomas } \\
\hline $\mathrm{RT}+$ chemo & Mut (+) & 27 & $9.3(4.3-\mathrm{NA})^{\mathrm{a}, \mathrm{b}}$ & $18.2(9.6-18.2)^{\mathrm{c}, \mathrm{d}}$ \\
\hline RT+chemo & Mut (-) & 15 & $2.3(0.6-7.0)^{\mathrm{a}-\mathrm{b}}$ & $8.0(3.8-8.3)^{\mathrm{c}, \mathrm{d}}$ \\
\hline RT only & Mut (+) & 7 & $3.1(0.7-4.3)^{\mathrm{b}}$ & $5.1(3.9-7.5)^{\mathrm{b}, \mathrm{d}, \mathrm{e}}$ \\
\hline RT only & Mut (-) & 9 & $1.9(0.4-4.2)$ & $3.1(0.5-4.2)^{\mathrm{b}, \mathrm{e}}$ \\
\hline \multicolumn{5}{|c|}{ Diffuse astrocytoma } \\
\hline $\mathrm{RT}+$ chemo & Mut (+) & 16 & $8.6(2.1-10.2)^{\mathrm{f}}$ & $\mathrm{NR}(6.3-\mathrm{NR})^{\mathrm{g}, \mathrm{h}}$ \\
\hline RT+chemo & Mut (-) & 13 & $1.8(0.6-4.5)^{\mathrm{f}}$ & $4.5(3.8-\mathrm{NR})^{\mathrm{g}, \mathrm{h}}$ \\
\hline RT only & Mut (+) & 3 & $3.1(0.7-3.1)^{\mathrm{f}}$ & $4.5(3.9-5.1)^{\mathrm{h}}$ \\
\hline RT only & Mut (-) & 8 & $2.4(0.5-\mathrm{NR})$ & $3.6(0.5-\mathrm{NR})$ \\
\hline \multicolumn{5}{|c|}{ Oligodendroglioma/oligoastrocytoma } \\
\hline $\mathrm{RT}+$ chemo & Mut (+) & 11 & $14.4(2.1-\mathrm{NR})^{\mathrm{e}}$ & $18.2(\mathrm{NR})^{\mathrm{a}}$ \\
\hline $\mathrm{RT}+$ chemo & Mut (-) & 2 & $8.1(\mathrm{NR})^{\mathrm{e}}$ & $8.1(\mathrm{NR})^{\mathrm{a}}$ \\
\hline RT only & Mut (+) & 4 & $3.7(1.0-4.3)^{\mathrm{e}, \mathrm{i}}$ & $6.3(5.1-7.5)^{\mathrm{a}, \mathrm{i}}$ \\
\hline RT only & Mut (-) & 1 & $0.4(\mathrm{NR})^{\mathrm{i}}$ & $1.6(\mathrm{NR})^{\mathrm{i}}$ \\
\hline
\end{tabular}

$\mathrm{NR}$, PFS or median survival time is not reached; CI, confident interval; RT, radiotherapy; chemo, chemotherapy; mut, mutation. ${ }^{\mathrm{a}} \mathrm{p}=0.02$; ${ }^{\mathrm{b}} \mathrm{p}=0.01 ;{ }^{\mathrm{c}} \mathrm{p}=0.004 ;{ }^{\mathrm{d}} \mathrm{p}=0.008 ;{ }^{\mathrm{e}} \mathrm{p}=0.03 ;{ }^{\mathrm{f}} \mathrm{p}=0.1 ;{ }^{\mathrm{g}} \mathrm{p}=0.06 ;{ }^{\mathrm{h}} \mathrm{p}=0.07 ;{ }^{\mathrm{i}} \mathrm{p}=0.05$.

Table V. Multivariate analyses of PFS and OS of patients with all grade II gliomas.

\begin{tabular}{|c|c|c|c|c|c|}
\hline Variable & $\begin{array}{l}\text { No. of } \\
\text { cases }\end{array}$ & $\begin{array}{l}\text { PFS hazard ratio } \\
\qquad(95 \% \mathrm{CI})\end{array}$ & $\begin{array}{l}\text { PFS p-value } \\
\text { (Cox) }\end{array}$ & $\begin{array}{l}\text { OS hazard ratio } \\
\quad(95 \% \mathrm{CI})\end{array}$ & $\begin{array}{l}\text { OS p-value } \\
\text { (Cox) }\end{array}$ \\
\hline \multicolumn{6}{|l|}{ Histology } \\
\hline Diffuse astrocytoma & 49 & 1 & 0.1 & 1 & 0.02 \\
\hline $\begin{array}{l}\text { Oligodendroglioma/ } \\
\text { oligoastrocytoma }\end{array}$ & 23 & $0.576(0.262-1.186)$ & & $0.290(0.086-0.815)$ & \\
\hline \multicolumn{6}{|l|}{$I D H$ mutation } \\
\hline Wild-type & 31 & 1 & 0.08 & 1 & 0.01 \\
\hline Mutation & 41 & $0.558(0.289-1.068)$ & & $0.365(0.155-0.819)$ & \\
\hline \multicolumn{6}{|l|}{ Age (years) } \\
\hline$\geq 40$ & 34 & 1 & 0.5 & 1 & 0.02 \\
\hline$<40$ & 38 & $0.802(0.440-1.460)$ & & $0.400(0.175-0.877)$ & \\
\hline \multicolumn{6}{|l|}{ Extent of removal } \\
\hline Partial removal and biopsy & 58 & 1 & 0.1 & 1 & 0.2 \\
\hline Total and subtotal removal & 14 & $0.556(0.222-1.217)$ & & $0.463(0.107-1.403)$ & \\
\hline \multicolumn{6}{|l|}{ Initial KPS } \\
\hline$<80$ & 4 & 1 & 0.01 & 1 & 0.0002 \\
\hline$\geq 80$ & 68 & $0.179(0.063-0.640)$ & & $0.045(0.011-0.198)$ & \\
\hline
\end{tabular}

CI, confident interval.

after surgery, those with $I D H 1 / 2$ mutations had significantly longer PFS and OS than those without IDHI/2 mutations (PFS: $\mathrm{p}=0.02$, OS: $\mathrm{p}=0.004$; Fig. $2 \mathrm{C}$ and D; Table IV).
An important finding is that the patients who were initially treated with chemoradiotherapy after surgery and had IDHI/2 mutations showed significantly longer PFS and OS than those 
Table VI. Multivariate analyses of PFS and OS of patients with all grade II gliomas with radiotherapy \pm chemotherapy.

\begin{tabular}{lcccc}
\hline Variable & $\begin{array}{c}\text { No. of } \\
\text { cases }\end{array}$ & $\begin{array}{c}\text { PFS hazard ratio } \\
(95 \% \mathrm{CI})\end{array}$ & $\begin{array}{c}\text { PFS p-value } \\
(\text { Cox })\end{array}$ & $\begin{array}{c}\text { OS hazard ratio } \\
(95 \% \mathrm{CI})\end{array}$ \\
\hline
\end{tabular}

\section{Histology}

Diffuse astrocytoma

Oligodendroglioma/

40

18

Oligoastrocytoma

\section{IDH mutation}

Wild-type
Mutation
Age (years)
$\geq 40$
$<40$

1

$0.758(0.362-1.559)$

Extent of removal

Partial removal and biopsy

Total and subtotal removal

$\begin{array}{cc}47 & 1 \\ 11 & 0.364(0.118-0.918)\end{array}$

Initial treatment

Radiotherapy alone

Chemoradiotherapy
16

42
1

$0.467(0.215-0.999)$

0.2 $0.549(0.209-1.290)$

0.05

$0.316(0.117-0.793)$

0.5

1

$0.745(0.300-1.808)$

0.03

1

0.08

$0.356(0.080-1.120)$

0.04
1

$0.198(0.073-0.529)$

CI, confident interval.

treated with radiotherapy alone with $I D H 1 / 2$ mutations. The PFS and OS of the patients with $I D H 1 / 2$ mutations who were initially treated with chemoradiotherapy after surgery $(n=27)$ were 9.3 and 18.2 years, respectively, and the PFS and OS of those treated with radiotherapy alone with $I D H 1 / 2$ mutations $(\mathrm{n}=7)$ were 3.1 and 5.1 years, respectively (PFS, $\mathrm{p}=0.01$; OS, $\mathrm{p}=0.008$ ). In the oligodendroglial tumors, the PFS and OS of the patients with $I D H 1 / 2$ mutations who were initially treated with chemoradiotherapy $(n=11)$ were 14.4 and 18.2 years, respectively, and the PFS and OS of those treated with radiotherapy alone with IDH1/2 mutations $(\mathrm{n}=4)$ were 3.7 and 6.3 years, respectively (PFS: $\mathrm{p}=0.03$, OS: $\mathrm{p}=0.02$; Fig. $2 \mathrm{G}$ and H). Similar tendencies, although not reaching statistical significance, were observed in the astrocytic tumors (PFS: $\mathrm{p}=0.1$, OS: $\mathrm{p}=0.07$; Fig. $2 \mathrm{E}$ and F).

The IDH1/2 status had no impact on the PFS of all the grade II glioma or diffuse astrocytoma patients who underwent radiotherapy alone. No significant difference in PFS was observed between the radiotherapy and chemoradiotherapy groups in the grade II glioma patients without $I D H 1 / 2$ mutations. Chemoradiotherapy did not prolong the PFS of the patients without IDHI/2 mutations in the astrocytic and oligodendroglial tumors.

Multivariate analysis. Oligodendroglial tumors (hazard ratio $(\mathrm{HR})=0.29, \mathrm{p}=0.02)$, age $<40$ years $(\mathrm{HR}=0.40, \mathrm{p}=0.02)$, initial KPS $\geq 80(\mathrm{HR}=0.045, \mathrm{p}=0.0002)$, and $I D H 1 / 2$ mutations $(\mathrm{HR}=0.37, \mathrm{p}=0.01)$ were favorable prognostic factors for OS time, as determined by the multivariate analysis, of the 72 patients included in the study (Table V). The IDH1/2 mutation status was not a prognostic factor for PFS when all the patients were considered, including those who did not undergo initial radiotherapy or chemotherapy $(\mathrm{p}=0.08)$. In contrast, total or subtotal tumor resection $(\mathrm{HR}=0.36, \mathrm{p}=0.03)$, chemoradiotherapy $(\mathrm{HR}=0.41, \mathrm{p}=0.04)$, and $I D H 1 / 2$ mutations $(\mathrm{HR}=0.47, \mathrm{p}=0.05)$ were favorable prognostic factors for PFS, as determined by the multivariate analysis, of the patients who were initially treated with radiotherapy or chemoradiotherapy (Table VI). Histological appearance was not a prognostic marker for PFS in this series $(\mathrm{p}=0.2)$ compared with $I D H 1 / 2$ mutations $(\mathrm{p}=0.05)$.

\section{Discussion}

WHO grade III and IV astrocytomas with IDH1/2 mutations have more favorable prognoses than those with wild-type IDHI/2 (17). IDH1/2 mutations, $1 \mathrm{p} / 19 \mathrm{q}$ codeletion, and $M G M T$ promoter methylation are pivotal prognostic factors in anaplastic oligodendroglial tumors treated with radiotherapy or chemoradiotherapy (EORTC 26951) (27). However, the impact of IDHI/2 mutations and/or $1 \mathrm{p} / 19 \mathrm{q}$ codeletion as biomarkers in grade II gliomas remains controversial. The present study was therefore aimed at identifying prognostic and/or predictive factors in grade II gliomas.

The presence of IDH1/2 mutations is a favorable prognostic marker for $O S$. The results of the univariate analysis revealed that the presence of $I D H 1 / 2$ mutations was a prognostic factor of longer OS $(\mathrm{p}=0.004)$ and PFS $(\mathrm{p}=0.04)$ in the entire patient cohort and among the patients who underwent with or without radiation therapy after initial surgery with or without chemotherapy. The multivariate analysis revealed that the presence of 
IDH1/2 mutations was associated with prolonged PFS $(\mathrm{p}=0.05)$ and OS ( $\mathrm{p}=0.01)$ in the patients who initially underwent radiotherapy with or without chemotherapy. Our results suggest that IDHI/2 mutations may be involved in the response to genotoxic therapy, such as radiotherapy or chemotherapy, and may act as a prognostic factor for chemotherapy or radiotherapy in grade II gliomas. There are currently increasing numbers of reports showing that $I D H 1 / 2$ mutations are prognostic markers for several malignancies, including grade II gliomas. Houillier et al (19) reported that the presence of IDHI/2 mutations is a significant prognostic marker for OS and chemosensitivity in low-grade glioma patients who were initially treated with temozolomide (TMZ) before any other treatment except surgery. Hartmann et al (16) reported that the IDHI mutation was a prognostic factor for PFS and OS in grade II glioma patients who underwent radiotherapy or chemotherapy after surgery. In our study, the presence of $I D H I / 2$ mutations was demonstrated by multivariate analysis to be a favorable prognostic factor $(\mathrm{p}=0.01)$ for OS but not a prognostic marker for PFS ( $\mathrm{p}=0.08$ ) in whole cohort, which included 14 patients who did not receive initial radiotherapy. Our finding that IDHI/2 status did not affect PFS was in line with the findings reported by Hartmann et al (16) or Houillier et al $(16,19)$, who showed that IDHI mutations did not affect the PFS in grade II glioma patients who did not receive radiotherapy or chemotherapy alone after surgery. Kim et al (21) and Mukasa et al (22) reported that the presence of $I D H I / 2$ mutations was not a prognostic factor for the survival of patients with low-grade glioma in univariate or multivariate analyses. The treatment of those patients was not fully described in their reports.

The presence of IDH1/2 mutations is a predictive marker for PFS in the grade II glioma patients treated with chemoradiotherapy. The patients who were initially treated with chemoradiotherapy after surgery showed significantly longer OS $(\mathrm{p}=0.0002)$ and PFS ( $p=0.01)$ than those treated with radiotherapy alone in our study. Chemoradiotherapy significantly prolonged PFS and OS compared with radiotherapy alone in all the grade II gliomas with $I D H 1 / 2$ mutations ( $\mathrm{p}=0.01$ and 0.0008 , respectively), diffuse astrocytoma ( $\mathrm{p}=0.1$ and 0.07 , respectively), and oligodendroglial tumors ( $\mathrm{p}=0.03$ and 0.02 , respectively) in the univariate analysis. Chemoradiotherapy was shown by multivariate analysis $(\mathrm{p}=0.04)$ to significantly prolong the PFS of grade II glioma patients carrying $I D H 1 / 2$ mutations who underwent radiotherapy with or without concomitant chemotherapy $(\mathrm{p}=0.04)$. In contrast, there were no differences in PFS between the radiotherapy and chemoradiotherapy groups among the grade II glioma patients without $I D H 1 / 2$ mutations in the univariate analysis. PFS did not differ by $I D H 1 / 2$ status in the grade II glioma patients who underwent radiotherapy alone. However, the present study was limited by the small number of samples and the differences in the follow-up periods between the radiation and chemoradiotherapy groups (4 and 7.6 years, respectively). A prospective study including a larger patient cohort is required to obtain conclusive evidence that the presence of $I D H 1 / 2$ mutations is a predictive marker for chemoradiotherapy in grade II gliomas. Nonetheless, our results suggest that $I D H 1 / 2$ mutation is a predictive marker for chemoradiotherapy in grade II glioma patients and indicate that these patients may benefit from concurrent chemotherapy and radiotherapy compared with patients who do not carry IDHI/2 mutations.
Mutations in IDHI/2 result in the acquisition of new enzymatic activity that enables the NADPH-dependent reduction of $\alpha$-ketoglutarate to 2-hydroxyglutarate, and the mutation confers oncogenic properties (28). IDHI mutations are early events in the development of astrocytomas and oligodendrogliomas (11). Another possible function of IDHI/2 mutations is the dominant-negative inhibition of the oxidative decarboxylation of isocitrate as a result of the formation of a wild-type/mutant heterodimer (29). Cellular IDH1 levels are associated with the protection from apoptosis and cell death after exposure to reactive oxygen species or ultraviolet B-induced phototoxicity and $I D H I / 2$ functions in cellular defense reactions (30). Glioma cells with $I D H 1 / 2$ mutations may be vulnerable to irradiation and chemotherapeutic agents, which might explain why $I D H 1 / 2$ mutations could be a predictive and prognostic marker for grade II gliomas in patients receiving chemoradiotherapy. Our findings warrant a prospective large-scale clinical study addressing the efficacy of chemoradiotherapy in grade II glioma patients in association with $I D H 1 / 2$ status.

Grade II glioma patients with wild-type IDH1/2 have poor prognoses even after total resection. The extent of resection of tumors has been reported to be significantly associated with survival and recurrence of disease in low-grade glioma patients $(9,31)$. In our study, the patients in the total or subtotal resection $(\geq 90 \%$ removal) group tended to have longer survival times than the patients in the partial $(<90 \%$ removal) or biopsy group $(\mathrm{p}=0.08)$. The patients without $I D H 1 / 2$ mutations had shorter OS than those with $I D H 1 / 2$ mutations in the total and subtotal resection groups $(\mathrm{p}=0.04)$ and in the partial and biopsy groups $(\mathrm{p}=0.01)$. Although the number of patients examined was small, we believe that this is a very important finding and that it indicates that patients without $I D H 1 / 2$ mutations may require more intensive treatment, such as chemoradiotherapy, even after total resection of the tumor.

$1 p / 19 q$ codeletion is not a prognostic factor. In our study, the OS and PFS in the diffuse astrocytomas with $1 \mathrm{p} / 19 \mathrm{q}$ codeletion tended to be longer than those in the patients without $1 \mathrm{p} / 19 \mathrm{q}$ codeletion, but the difference did not reach statistical significance. Furthermore, no significant differences were observed between the grade II glioma patients with regard to $1 \mathrm{p} / 19 \mathrm{q}$ status. Prior studies reported that the presence of the $1 \mathrm{p} / 19 \mathrm{q}$ codeletion was significantly associated with longer OS in lowgrade gliomas $(12,13,15,21,32)$. On the other hand, Houillier et al and Mukasa et al $(19,22)$ reported that loss of $1 \mathrm{p} / 19 \mathrm{q}$ was not a sensitive prognostic biomarker. Ichimura et al and Vogazianou et al reported that total 1p/19q loss is rare and that when present, it is associated with longer survival than other $1 p / 19 q$ changes in adult gliomas independent of pathological diagnosis $(14,15)$. Deletion of $1 p$ or $19 q$ was determined mainly by FISH analysis in our study, and this technique cannot discriminate between total and partial $1 \mathrm{p} / 19 \mathrm{q}$ deletion, which might explain the discrepancy in the results.

Clinicopathological factors in grade II gliomas. The multivariate analysis showed that age $\geq 40$ years $(\mathrm{p}=0.02)$, astrocytic tumors ( $\mathrm{p}=0.02)$, initial KPS $<80(\mathrm{p}=0.0002)$, and wild-type $I D H 1 / 2(\mathrm{p}=0.01)$ were unfavorable prognostic factors in our series. These results are generally in line with previous reports 
showing that older age, astrocytic histology, presence of neurologic deficits before surgery, largest tumor diameter, and tumors crossing the midline were important unfavorable prognostic factors for survival in adult patients with low-grade gliomas (5-9).

In conclusion, the multivariate analysis showed that age $<40$ years, oligodendroglial tumors, initial KPS $\geq 80$, and $I D H 1 / 2$ mutations were favorable prognostic factors for survival of the grade II glioma patients. The presence of $I D H 1 / 2$ mutations was a prognostic factor for grade II glioma patients with radiotherapy. Furthermore, it is a predictive factor of response to chemoradiotherapy in grade II gliomas. Patients carrying $I D H 1 / 2$ mutations may benefit more from concurrent chemotherapy and radiotherapy compared with those without $I D H 1 / 2$ mutations.

\section{Acknowledgements}

We thank all the doctors, nurses and medical staff in National Cancer Center Hospital who attended to the glioma patients in these 20 years. This study was supported by National Cancer Center Research and Development Fund no. 23-A-49.

\section{References}

1. Louis DN, Ohgaki H, Wiestler OD, Cavenee WK, Burger PC, Jouvet A, Scheithauer BW and Kleihues P: The 2007 WHO classification of tumours of the central nervous system. Acta Neuropathol 114: 97-109, 2007.

2. Bauman G, Fisher B, Watling C, Cairncross JG and Macdonald D: Adult supratentorial low-grade glioma: long-term experience at a single institution. Int J Radiat Oncol Biol Phys 75: 1401-1407, 2009.

3. van den Bent MJ, Afra D, de Witte O, Ben Hassel M, Schraub S, Hoang-Xuan K, Malmstrom PO, Collette L, Pierart M, Mirimanoff R and Karim AB: Long-term efficacy of early versus delayed radiotherapy for low-grade astrocytoma and oligodendroglioma in adults: the EORTC 22845 randomised trial. Lancet 366: 985-990, 2005.

4. Shaw EG, Wang M, Coons S, Brachman D, Buckner JC, Stelzer K, Barger G, Brown PD, Gilbert MR and Mehta MP. Final report of Radiation Therapy Oncology Group (RTOG) protocol 9802: radiation therapy (RT) versus $\mathrm{RT}+$ procarbazine, CCNU, and vincristine (PCV) chemotherapy for adult low-grade glioma (LGG). J Clin Oncol (ASCO Meeting abst) 26: 2006, 2008

5. Bauman G, Lote K, Larson D, Stalpers L, Leighton C, Fisher B, Wara W, MacDonald D, Stitt L and Cairncross JG: Pretreatment factors predict overall survival for patients with low-grade glioma: a recursive partitioning analysis. Int J Radiat Oncol Biol Phys 45: 923-929, 1999.

6. Chang EF, Smith JS, Chang SM, Lamborn KR, Prados MD, Butowski N, Barbaro NM, Parsa AT, Berger MS and McDermott MM: Preoperative prognostic classification system for hemispheric low-grade gliomas in adults. J Neurosurg 109: 817-824, 2008

7. Pignatti F, van den Bent M, Curran D, Debruyne C, Sylvester R, Therasse P, Afra D, Cornu P, Bolla M, Vecht C and Karim AB: Prognostic factors for survival in adult patients with cerebral low-grade glioma. J Clin Oncol 20: 2076-2084, 2002.

8. Schiff D, Brown PD and Giannini C: Outcome in adult low-grade glioma: the impact of prognostic factors and treatment. Neurology 69: 1366-1373, 2007.

9. Smith JS, Chang EF, Lamborn KR, Chang SM, Prados MD, Cha S, Tihan T, Vandenberg S, McDermott MW and Berger MS: Role of extent of resection in the long-term outcome of low-grade hemispheric gliomas. J Clin Oncol 26: 1338-1345, 2008.

10. Smith JS, Alderete B, Minn Y, Borell TJ, Perry A, Mohapatra G, Hosek SM, Kimmel D, O'Fallon J, Yates A, et al: Localization of common deletion regions on $1 \mathrm{p}$ and $19 \mathrm{q}$ in human gliomas and their association with histological subtype. Oncogene 18: 4144-4152, 1999.
11. Watanabe T, Nobusawa S, Kleihues $P$ and Ohgaki H: IDH1 mutations are early events in the development of astrocytomas and oligodendrogliomas. Am J Pathol 174: 1149-1153, 2009.

12. Smith JS, Perry A, Borell TJ, Lee HK, O'Fallon J, Hosek SM, Kimmel D, Yates A, Burger PC, Scheithauer BW and Jenkins RB: Alterations of chromosome arms $1 \mathrm{p}$ and $19 \mathrm{q}$ as predictors of survival in oligodendrogliomas, astrocytomas, and mixed oligoastrocytomas. J Clin Oncol 18: 636-645, 2000.

13. Mariani L, Deiana G, Vassella E, Fathi AR, Murtin C, Arnold M, Vajtai I, Weis J, Siegenthaler P, Schobesberger M and Reinert MM: Loss of heterozygosity $1 \mathrm{p} 36$ and $19 \mathrm{q} 13$ is a prognostic factor for overall survival in patients with diffuse WHO grade 2 gliomas treated without chemotherapy. J Clin Oncol 24: 4758-4763, 2006.

14. Ichimura K, Vogazianou AP, Liu L, Pearson DM, Backlund LM, Plant K, Baird K, Langford CF, Gregory SG and Collins VP: 1p36 is a preferential target of chromosome 1 deletions in astrocytic tumours and homozygously deleted in a subset of glioblastomas. Oncogene 27: 2097-2108, 2008.

15. Vogazianou AP, Chan R, Backlund LM, Pearson DM, Liu L, Langford CF, Gregory SG, Collins VP and Ichimura K: Distinct patterns of $1 p$ and $19 q$ alterations identify subtypes of human gliomas that have different prognoses. Neurooncology 12: 664-678, 2010

16. Hartmann C, Hentschel B, Tatagiba M, Schramm J, Schnell O, Seidel C, Stein R, Reifenberger G, Pietsch T, von Deimling A, Loeffler $\mathrm{M}$ and Weller M: Molecular markers in low-grade gliomas: predictive or prognostic? Clin Cancer Res 17: 4588-4599, 2011.

17. Yan H, Parsons DW, Jin G, McLendon R, Rasheed BA, Yuan W, Kos I, Batinic-Haberle I, Jones S, Riggins GJ, et al: IDH1 and IDH2 mutations in gliomas. N Engl J Med 360: 765-773, 2009.

18. Balss J, Meyer J, Mueller W, Korshunov A, Hartmann C and von Deimling A: Analysis of the IDH1 codon 132 mutation in brain tumors. Acta Neuropathol 116: 597-602, 2008.

19. Houillier C, Wang X, Kaloshi G, Mokhtari K, Guillevin R, Laffaire J, Paris S, Boisselier B, Idbaih A, Laigle-Donadey F, et al: IDH1 or IDH2 mutations predict longer survival and response to temozolomide in low-grade gliomas. Neurology 75: 1560-1566, 2010.

20. Metellus P, Coulibaly B, Colin C, de Paula AM, Vasiljevic A, Taieb D, Barlier A, Boisselier B, Mokhtari K, Wang XW, et al: Absence of IDH mutation identifies a novel radiologic and molecular subtype of WHO grade II gliomas with dismal prognosis. Acta Neuropathol 120: 719-729, 2010.

21. Kim YH, Nobusawa S, Mittelbronn M, Paulus W, Brokinkel B, Keyvani K, Sure U, Wrede K, Nakazato Y, Tanaka Y, et al: Molecular classification of low-grade diffuse gliomas. Am J Pathol 177: 2708-2714, 2010.

22. Mukasa A, Takayanagi S, Saito K, Shibahara J, Tabei Y, Furuya K, Ide T, Narita Y, Nishikawa R, Ueki K and Saito N: Significance of IDH mutations varies with tumor histology, grade, and genetics in Japanese glioma patients. Cancer Sci 103: 587-592, 2012.

23. Camelo-Piragua S, Jansen M, Ganguly A, Kim JC, Louis DN and Nutt CL: Mutant IDH1-specific immunohistochemistry distinguishes diffuse astrocytoma from astrocytosis. Acta Neuropathol 119: 509-511, 2010.

24. Hartmann C, Meyer J, Balss J, Capper D, Mueller W, Christians A, Felsberg J, Wolter M, Mawrin C, Wick W, et al: Type and frequency of IDH1 and IDH2 mutations are related to astrocytic and oligodendroglial differentiation and age: a study of 1,010 diffuse gliomas. Acta Neuropathol 118: 469-474, 2009.

25. Franco-Hernandez C, Martinez-Glez V, de Campos JM, Isla A, Vaquero J, Gutierrez M, Casartelli C and Rey JA: Allelic status of $1 \mathrm{p}$ and $19 \mathrm{q}$ in oligodendrogliomas and glioblastomas: multiplex ligation-dependent probe amplification versus loss of heterozygosity. Cancer Genet Cytogenet 190: 93-96, 2009.

26. Schouten JP, McElgunn CJ, Waaijer R, Zwijnenburg D, Diepvens F and Pals G: Relative quantification of 40 nucleic acid sequences by multiplex ligation-dependent probe amplification. Nucleic Acids Res 30: e57, 2002.

27. van den Bent MJ, Dubbink HJ, Marie Y, Brandes AA, Taphoorn MJ, Wesseling P, Frenay M, Tijssen CC, Lacombe D, Idbaih A, et al: IDH1 and IDH2 mutations are prognostic but not predictive for outcome in anaplastic oligodendroglial tumors: a report of the European Organization for Research and Treatment of Cancer Brain Tumor Group. Clin Cancer Res 16: 1597-1604, 2010 . 
28. Dang L, White DW, Gross S, Bennett BD, Bittinger MA, Driggers EM, Fantin VR, Jang HG, Jin S, Keenan MC, et al: Cancer-associated IDH1 mutations produce 2-hydroxyglutarate. Nature 462: 739-744, 2009.

29. Zhao S, Lin Y, Xu W, Jiang W, Zha Z, Wang P, Yu W, Li Z, Gong L, Peng Y, et al: Glioma-derived mutations in IDH1 dominantly inhibit IDH1 catalytic activity and induce HIF-1alpha. Science 324: 261-265, 2009.

30. Reitman ZJ and Yan H: Isocitrate dehydrogenase 1 and 2 mutations in cancer: alterations at a crossroads of cellular metabolism. J Natl Cancer Inst 102: 932-941, 2010.
31. Ahmadi R, Dictus C,Hartmann C, Zurn O, Edler L, Hartmann M, Combs S, Herold-Mende C, Wirtz CR and Unterberg A: Long-term outcome and survival of surgically treated supratentorial low-grade glioma in adult patients. Acta Neurochir 151: 1359-1365, 2009.

32. Daniels TB, Brown PD, Felten SJ, Wu W, Buckner JC, Arusell RM, Curran WJ, Abrams RA, Schiff D and Shaw EG: Validation of EORTC prognostic factors for adults with low-grade glioma: a report using intergroup 86-72-51. Int J Radiat Oncol Biol Phys 81: 218-224, 2011. 